\title{
Ciclo de Mejora Docente en el Aula aplicado a la asignatura de Arte Universal en el Grado en Historia (Universidad de Sevilla)
}

\section{Teaching Improvement Cycle applied to the subject of Universal Art. Degree in History (University of Seville)}

CARMEn Vallejo NaRANJO

ORCID: https://orcid.org/0000-0002-3154-1956

Universidad de Sevilla

Departamento de Historia del Arte

cvallejo@us.es

DOI: http://dx.doi.org/10.12795/9788447231003.067

Pp.: 1412-1435 


\section{Introducción}

El Ciclo de Mejora en el Aula (CIMA) fue aplicado en el tema 3, Arte griego, de la asignatura obligatoria de segundo curso, Arte Universal, correspondiente al Grado de Historia que se imparte en el primer cuatrimestre de lunes a jueves en horario de 20 a 21 h. La realización del CIMA se ha producido completamente en línea a través de la plataforma de enseñanza virtual. Obviamente, en esta situación extraordinaria, se adaptó el diseño de las secuencias de actividades, contando con el contacto a distancia y la herramienta digital, que como tal constituye un recurso, pero en ningún caso es el factor desencadenante del cambio, pues el aprendizaje se realiza en un espacio tanto fisico como virtual (Porlán, 2020a).

\section{Diseño previo del Ciclo de Mejora en el Aula}

\section{Mapa de Contenidos}

El mapa se realiza sobre los contenidos del Arte prehelénico: Creta y Micenas. Así como de los tres periodos griegos: arcaico, clásico y postclásico.

El mapa se realiza de un modo holístico, es decir, absolutamente transversal, tanto en la naturaleza de los contenidos (conceptuales, metodológicos, actitudinales, etc.) como en su aplicación multidisciplinar (Arquitectura, Pintura, Escultura y Artes Suntuarias). Se tomó como modelo, el propio proceso de comunicación o expresión humana en relación a su medio espacio-temporal. Dado que el primigenio medio de expresión manual con la que el hombre refleja su cosmovisión es el registro plástico, muy anterior al escrito que marca el inicio de la historia. El mapa de contenidos refleja esa necesidad nuclear y global del ser humano en la comunicación con su medio como una onda expansiva que se sucede cronológicamente en una constante tanto diacrónica o evolutiva como sincrónica. Con Ciclos de Mejora en el Aula (2020). Experiencias de Innovación Docente de la US
Esta obra se distribuye con la licencia Creative Commons 
claros hitos históricos que causan cambios en las mentalidades colectivas y marcan el punto de inflexión en la transición, tanto hacia los sucesivos momentos de plenitud de los estilos, como de la disolución de los mismos en otros nuevos. Al igual que sucede con nuestro propio sistema de ideas que permanece, se desarrolla y reconstruye (Porlán, 2020a). Con esta constante humanística, se plantea el tema, partiendo de un contexto físico e histórico-cultural que aporta génesis griega al factor oriental del que se nutre el Mediterráneo. He tejido un hilo de Ariadna con la selección de los datos y contenidos conceptuales, transversales, procedimentales y actitudinales (Porlán, 2019) y las obras en la que estos se van a verificar. Lo que supone un proceso de aprendizaje tanto conceptual como metodológico de la propia disciplina. El mapa refleja cómo, en las nuevas coordenadas espacio-temporales y socio-culturales, la herencia artística recibida va a inocular en este contexto geográfico un nuevo estilo, que en un proceso "biológico" de maduración (inicial, pleno y tardío) estará siempre influenciado por constantes transversales como la iconografía-iconología, la composición, las técnicas y el estilo que, como una hélice, baten por igual a las cuatro disciplinas y generan una sola expresión artística y estética de conjunto, característica de un territorio y un período concreto de la que recibe su designación genérica. Con esta dinámica de contenidos y procedimientos, articulados a través de las grandes preguntas clave, el alumno recibe un sistema organizador que le permitirá adquirir los mecanismos y asimilar las constantes de un esquema de producción que le dará las claves en un aprendizaje del tema y de la asignatura profundo y sistemático (Porlán, 2020a). Comprendiendo qué es el arte, cuándo y cómo se articula el bagaje intelectual e ideológico que lo define, por qué y para qué se crea artísticamente. Aprendiendo a reconocer su linaje artístico en los antecedentes de los que parte y a valorar en los temas sucesivos la trascendencia de su proyección en la historia, sin tener que realizar un inabarcable ejercicio memorístico que lo aleja del disfrute de las cosas bellas y los hitos humanos.

Ciclos de Mejora en el Aula (2020). Experiencias de Innovación Docente de la US Esta obra se distribuye con la licencia Creative Commons 


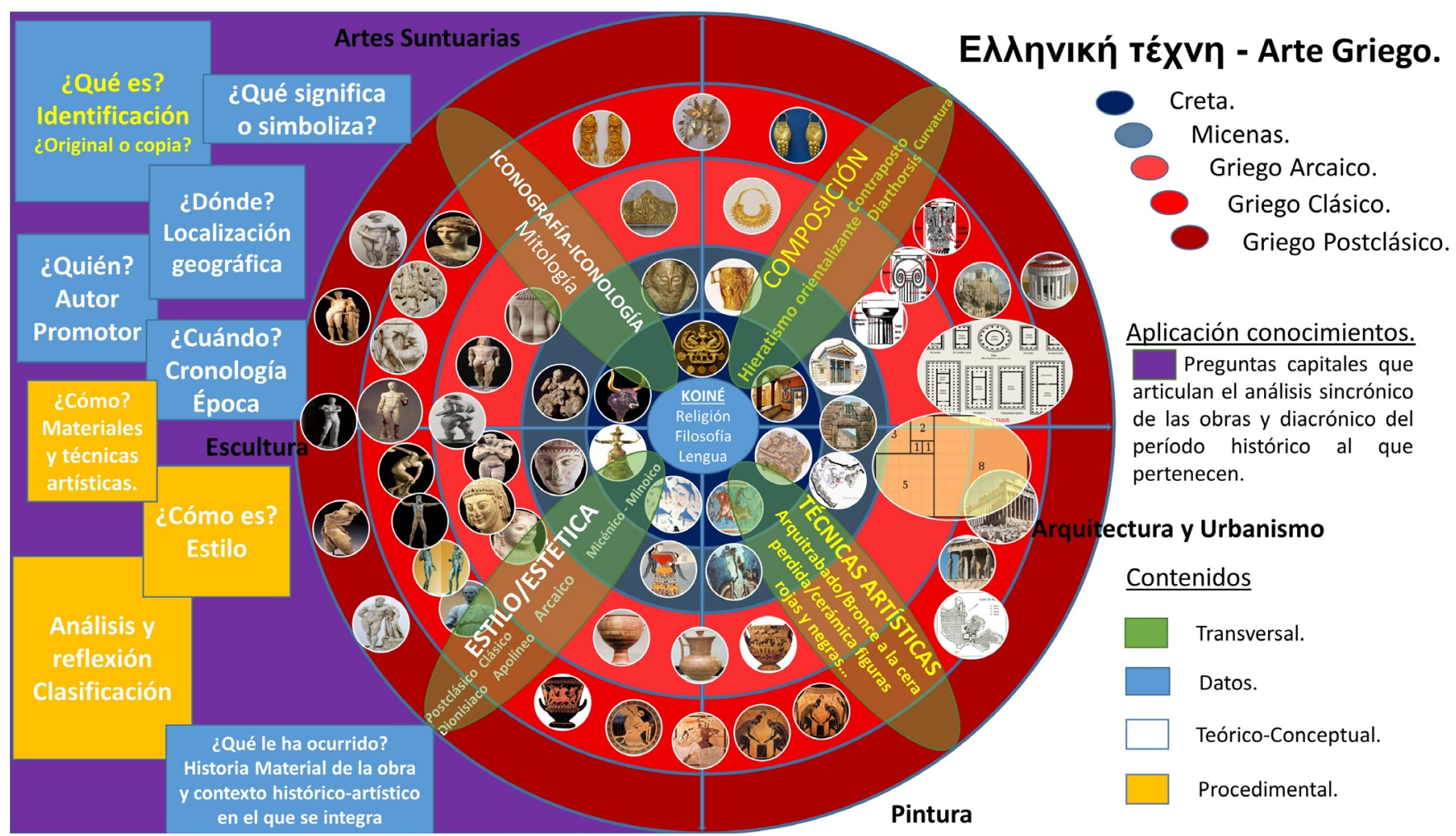

Figura 1. Esquema del Mapa de Contenidos y Preguntas clave.

Ciclos de Mejora en el Aula (2020). Experiencias de Innovación Docente de la US (c) E $\ominus$ Esta obra se distribuye con la licencia Creative Commons Internacional (CC BY-NC-ND 4.0.) 


\section{Modelo metodológico posible}

A través del curso he podido comprobar que los recursos innatos o adquiridos en nuestra experiencia no son suficientes para perfeccionar un modelo docente que requiere de una metodología didáctica científica y precisa para cada programa curricular (Porlán, 2020b). Dirigir al alumno en su aprendizaje hacia el conocimiento, a través de su propia experiencia y necesidad vital, para que elaborare su estructura cognitiva, es el objetivo principal. Todo ello, necesita de un potente repertorio de estrategias didácticas que incentiven, estimulen y comprometan al alumno con su propio proceso de aprendizaje y que deben estar previamente diseñadas en función del objetivo principal mencionado y de los distintos objetivos transversales a conseguir (Porlán, 2019).

Por todo ello, la secuencia de actividades programadas que pretendo aplicar es un modelo docente intermedio que baraja las metodologías transmisiva y constructivista (Vygotsky, 2003; Piaget, 1970 y Bruner, 1978) para fomentar la participación del alumno en clase y cultivar la curiosidad que lo ponga en movimiento a través de un repertorio variado de actividades:

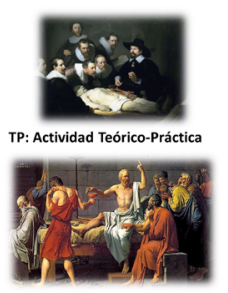

Pr: Problemas

P: Actividad teórico-práctica sobre un caso concreto.

le: Ideas de las que parten los alumnos.

Ac: Actividades de Contraste: Debate, Nuevos datos, reelaboración de planteamientos.

T: Teoría interactiva: Clase magistral o transmisiva con preguntas y planteamientos del profesor.

Pr: Introducción de un problema o dilema.

c: Conclusiones y consolidación de los conocimientos adquiridos.

Figura 2. Leyenda actividades modelo metodológico.

Ciclos de Mejora en el Aula (2020). Experiencias de Innovación Docente de la US Esta obra se distribuye con la licencia Creative Commons 
He combinado dos tipologías básicas que entiendo se complementan. El primer submodelo se resuelve de modo polivalente y flexible en el ritmo, intensidad y dirección de aplicación de las actividades referidas (Figura 2). Esto es, más plástica didácticamente hablando, en relación al contexto del grupo y a la naturaleza de los contenidos. Controlando todos estos factores para no presentar un patrón reconocible del método que desacelere la inercia participativa. Alternando o interactuando con el primero se complementa el proceso del segundo submodelo que refleja un total apoyo de la interactuación y protagonismo de los alumnos a través de la exposición de un trabajo previo y su exposición sobre un contenido concreto, realizado de modo individual o en grupo, y su posterior debate que ejercite y promueva el razonamiento crítico de su propio conocimiento adquirido.

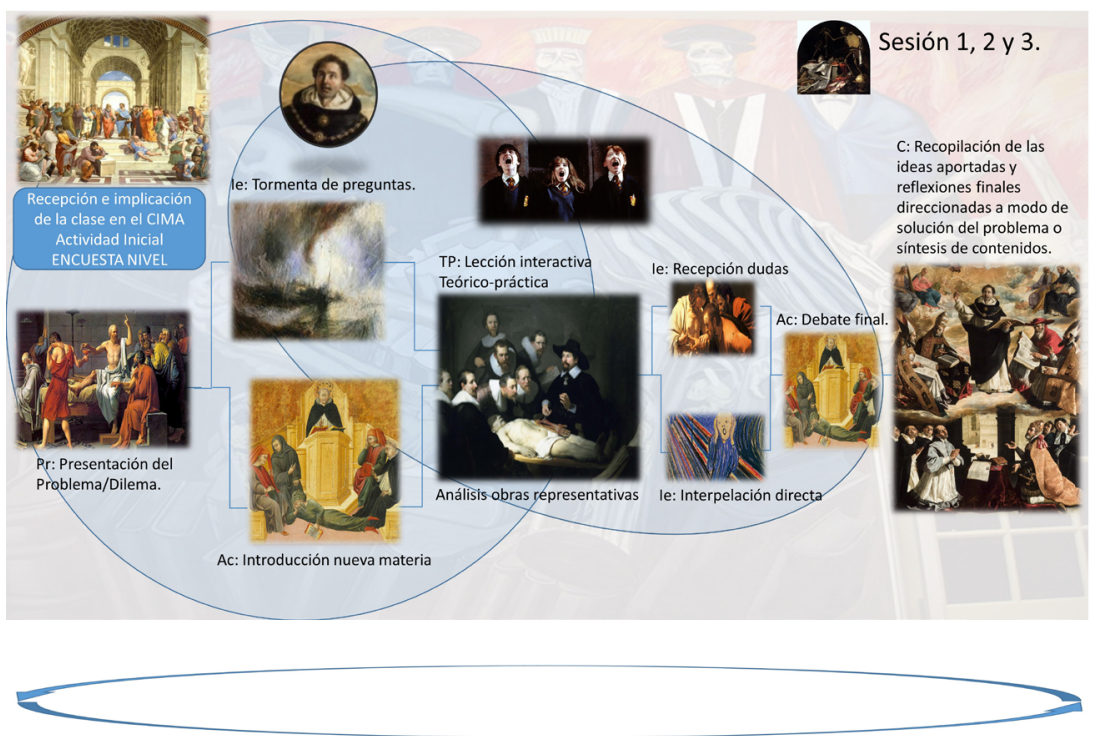

Ciclos de Mejora en el Aula (2020). Experiencias de Innovación Docente de la US Esta obra se distribuye con la licencia Creative Commons Reconocimiento-NoComercial-SinObraDerivada Internacional (CC BY-NC-ND 4.0.) 


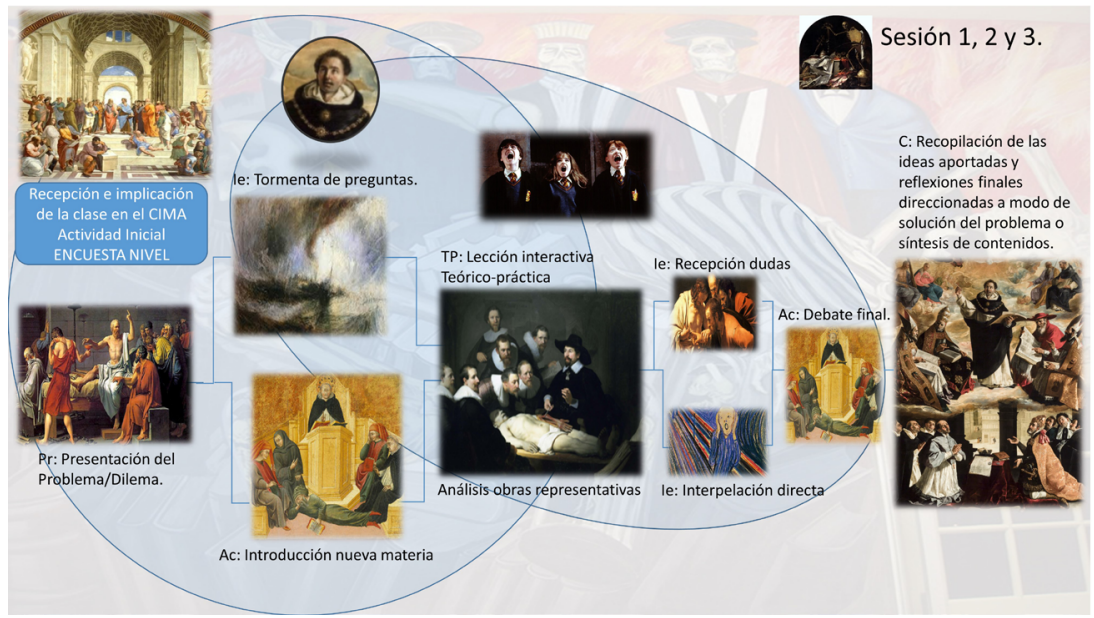

Figura 3. Modelo metodológico.

\section{Secuencia de actividades programadas.}

El diseño de la secuencia de actividades articula la aplicación del modelo metodológico con la proporción de un modelo intermedio durante tres sesiones consecutivas dirigidas por el profesor a través de planteamiento de problemas y actividades de contraste por una sesión puramente constructivista donde el alumnado asume la responsabilidad individual y colectiva del acceso, transmisión y análisis conclusivo del tema propuesto con la moderación del profesor que, en las ocho horas de este CIMA, se debe aplicar de forma completa dos veces.

Tabla 1 - Secuencia de actividades de la sesión primera

\begin{tabular}{|l|l|l|l|}
\hline \multicolumn{4}{|c|}{ Sesión 1 - La Hélade y su cultura. } \\
\hline Act. $\mathbf{0}$ & $\begin{array}{l}\text { Recepción del cuestionario y bienvenida al ejercicio del } \\
\text { CIMA 2. }\end{array}$ & $\mathbf{2}^{\prime}$ & le \\
\hline $\begin{array}{l}\text { Agradecimiento por la participación en el cuestionario inicial y compromiso } \\
\text { con el proyecto. } \\
\text { Objetivo: Asumir el nivel de conocimiento sobre el arte griego del } \\
\text { alumnado, ajustar el punto de partida de los contenidos y seleccionar los } \\
\text { absolutamente necesarios en la superación del aprendizaje. }\end{array}$ & $\mathbf{1 0}$ & PR \\
\hline
\end{tabular}

Ciclos de Mejora en el Aula (2020). Experiencias de Innovación Docente de la US CC $\Theta$ Esta obra se distribuye con la licencia Creative Commons Reconocimiento-NoComercial-SinObraDerivada $\quad 4.0$ Internacional (CC BY-NC-ND 4.0.) 
¿Qué es Europa? (juego de palabras entre el nombre de nuestro continente y la princesa raptada por Zeus metamorfoseado en Toro). Referencia de la cultura creto-minoica con la que empezaremos. ¿Qué es la cultura europea? ¿Reconocemos nuestra genealogía cultural? ¿Hay dos Europas?

Objetivo: Reflexión sobre nuestra propia identidad cultural en la que a partir de este tema nos vamos a instalar durante el curso. Las dos grandes identidades del legado antiguo: nórdico y mediterráneo.

\begin{tabular}{|l|l|l|l|}
\hline Act. $\mathbf{2}$ & ¿Y tú, de quién eres? & $\mathbf{1 0}^{\prime}$ & Ac \\
\hline
\end{tabular}

En relación a la participación y respuestas dadas, activaremos el debate con una tormenta de preguntas dirigidas a conocer la percepción del alumnado sobre el mosaico cultural europeo: ¿Cómo reflexionas sobre tu cultura, lo has hecho alguna vez? ¿De dónde procedes? ¿A qué cultura crees que perteneces? ¿Te reconoces en la cultura mediterránea, por qué?

Objetivo: Georreferenciación de nuestra herencia cultural.

\begin{tabular}{l|l|l|l|}
\hline Act. 3 & $\begin{array}{l}\text { ¿Qué es el arte griego antiguo? ¿Cómo es? ¿Cuánto } \\
\text { abarca? }\end{array}$ & $\mathbf{1 0}$ & Ac \\
\hline $\begin{array}{l}\text { Visualización y comentario sobre una secuencia de obras griegas que } \\
\text { presentan una visión general de los distintos períodos y producciones }\end{array}$ \\
$\begin{array}{l}\text { artísticas que vamos a estudiar. } \\
\text { Objetivo: Contextualización para el reconocimiento de los factores estéticos } \\
\text { de los distintos períodos }\end{array}$
\end{tabular}

\begin{tabular}{|l|l|l|l|}
\hline Act. $\mathbf{4}$ & ¿Qué une a todas estas culturas? & $\mathbf{1 0}$ & TP \\
\hline
\end{tabular}

¿Quién me puede presentar a un@ dios@grieg@? ¿Cuáles son las letras griegas más familiares para vosotros? Presentación de los elementos que componen la Koiné: Religión. Filosofía, Lengua.

Objetivo: Conocimiento de los pilares de la cultura griega que contienen el bagaje intelectual de la producción artística y reconocimiento de aquellos que perduran entre nosotros.

\begin{tabular}{l|l|l|l|}
\hline Act. $\mathbf{5}$ & Resolución de dudas espontáneas. & $\mathbf{5}^{\prime}$ & le \\
\hline $\begin{array}{l}\mid l \\
\text { Objetivo: Participación, aclaración de contenidos y oportunidad de seguir } \\
\text { profundizando en el tema. }\end{array}$ \\
\hline
\end{tabular}

\begin{tabular}{|l|l|l|l|}
\hline Act. $\mathbf{6}$ & Interpelación directa a los alumnos. & $\mathbf{5}^{\prime}$ & le \\
\hline
\end{tabular}

Objetivo: Fomentar, en caso de baja cooperación, la actitud de participación.

\begin{tabular}{|l|l|l|l|}
\hline Act. 7 & Reordenación de las ideas aportadas y reflexión final. & 10' & C \\
\hline Recopilación, ordenación e inclusión si faltara alguna de las ideas nucleares \\
y reflexiones finales direccionadas a la resolución del problema presentado: \\
Somos y pertenecemos a la Europa mediterránea e hijos de la Grecia Antigua. \\
Objetivo: Definición de una imagen mental concreta del mundo griego \\
antiguo y su cultura. Donde, ya debidamente contextualizados, vamos a \\
avanzar a través de su producción artística.
\end{tabular}

Ciclos de Mejora en el Aula (2020). Experiencias de Innovación Docente de la US Esta obra se distribuye con la licencia Creative Commons Reconocimiento-NoComercial-SinObraDerivada $\quad 4.0$ Internacional (CC BY-NC-ND 4.0.) 
Tabla 2 - Secuencia de actividades de la sesión segunda

Sesión 2 - El arte de las culturas prehelénicas: Creta y Micenas.

\begin{tabular}{|l|l|l|l|}
\hline Act. 1 & ¿Cómo persiste una impronta cultural? & $5^{\prime}$ & PR \\
\hline
\end{tabular}

¿Cuál sería el icono que identifica España? El Toro de Osborne ¿Por qué? ¿Nos representa a todos los españoles? ¿A qué ciclo ceremonial ya estudiado responde? Comenzamos con: Creta.

Objetivos: Presentación de la Hélade como madre de la cultura mediterránea. Presentación de los dos periodos prehelénicos.

\begin{tabular}{|l|l|l|l|}
\hline Act. 2 & Talasocracia - Geografia estratégica. & $\mathbf{1 0}^{\prime}$ & Ac \\
\hline Gran tormenta de ideas/preguntas dirigidas y secuenciadas: ¿Cómo es \\
posible que un pequeño territorio enclavado en el eje equidistante de \\
todo el Mediterráneo, con una economía talasocrática no sea hostigado y \\
mantenga una cultura conceptualmente "arcádica"? Solicitud de ejemplos. \\
Objetivo: Contextualización de las referencias cronológicas, físicas, sociales, \\
económicas y administrativas de la civilización cretense.
\end{tabular}

\begin{tabular}{|l|l|l|l|}
\hline Act. 3 & $\begin{array}{l}\text { Mitologia e Historia: Teseo y el Minotauro . Contexto } \\
\text { Creta antigua. }\end{array}$ & $\mathbf{1 0}^{\prime}$ & $\mathbf{T}$ \\
\hline
\end{tabular}

Presentación/explicación del contexto mitológico del mundo creto-minoico: rey Minos. Contexto geográfico y socio-económico de la isla y organización territorial. Palacio de Cnosos como paradigma de su cultura.

Objetivo: Explicación del contexto que define y contiene la producción artística que vamos a estudiar.
\begin{tabular}{|l|l|l|l|}
\hline Act. 4 & Análisis teórico-práctico de las principales obras que & 15' & TP
\end{tabular} representan y definen el arte creto-minoico.
¿Cuáles son las obras Creto-minoicas, cómo son, dónde están, quién las hizo, cómo se hicieron, qué función tienen, qué significan, qué estética presentan? Objetivo: Aprendizaje a través del análisis directo de las principales obras cretominoicas.

\begin{tabular}{l|l|l|l|}
\hline Act. $\mathbf{5}$ & Arte Micénico ¡Houston, tenemos un problema! & $\mathbf{5}^{\prime}$ & $\mathbf{A c}$ \\
\hline \multicolumn{4}{l}{ Confrontación entre oferentes del Palacio de Cnosos y del Palacio de Tirinto: } \\
¿Cómo distinguir la pintura Minoica de la Micénica? Mismo estilo, distinto \\
tratamiento plástico y temático. \\
Objetivos: Aprender a ver. Necesidad de aplicar las preguntas nucleares. \\
$\begin{array}{l}\text { Fijación del practicum de la disciplina y de la propia práctica de la evaluación. } \\
\text { Evitar memorizar los contenidos metodológicos. }\end{array}$ \\
\hline Act. $\mathbf{6}$ & Aculturación del arte micénico. & $\mathbf{1 5}$ & TP \\
\hline
\end{tabular}
Ciclos de Mejora en el Aula (2020). Experiencias de Innovación Docente de la US
Esta obra se distribuye con la licencia Creative Commons 
A través del conocimiento del arte cretense que rige hegemónico en la Península Egea, se analizaron los préstamos creados en Micenas. Ahora destacamos sus diferencias: Murallas de Micenas y Tesoro de Atreo y reubicamos los espacios comunes: Palacio y Megarón de Tirinto donde vimos su pintura.

Objetivos: Concluir con una imagen global y diferenciada de la producción de cada estilo Prehelénico.

Tabla 3 - Secuencia de actividades de la sesión tercera

\begin{tabular}{|c|c|c|c|}
\hline \multicolumn{4}{|c|}{ Sesión 3 - Grecia. } \\
\hline Act. 1 & ¡Ha nacido “Grecia”! & $20^{\prime}$ & TP \\
\hline \multicolumn{4}{|c|}{$\begin{array}{l}\text { Reubicación y lanzamiento, a través de una perspectiva general, del repertorio } \\
\text { artístico más representativo en el que nos introducimos: Arcaico, Clásico y } \\
\text { Postclásico. Secuencialización desde los núcleos de gestación prehelénicos } \\
\text { y de los grandes paradigmas de la cultura griega: Urbanismo y arquitectura: } \\
\text { Polis (Mileto), Templo (cánones: dórico, jónico, corintio y número áureo). } \\
\text { Escultura y pintura como expresión religiosa y moral que canaliza el discurso } \\
\text { político con la presentación de los principales ejemplares. } \\
\text { Objetivo: Amplia presentación teórico-práctica que instruya al alumno en } \\
\text { los contenidos transversales básicos. Adquisición de glosario procedimental. }\end{array}$} \\
\hline
\end{tabular}

Act. 2 Interpelación directa.

\begin{tabular}{|l|l|l}
\hline & le \\
\hline & & -10
\end{tabular}

Objetivo: Asegurar que los grandes pilares que sustentan esta estructura han sido asimilados para poder avanzar con seguridad en los siguientes apartados.

\begin{tabular}{|l|l|l|l|}
\hline Act. 3 & Período Arcaico, ss. VII-VI & $\mathbf{1 5}^{\prime}$ & $\mathbf{T}$ \\
\hline
\end{tabular}

Período arcaico como transición próximo-oriental, trasladada técnica y estéticamente a través de la cultura prehelénica. Arcaico Antiguo, Pleno y Final. Arquitectura: Aplicación de los elementos señalados en la planta y alzado del Templo Griego arcaico, los cánones correspondientes a este período: dórico, jónico y cariático. Escultura: Relieve arcaico. Se analizan sobre: Templo de Artemisa en Corfú.

Objetivos: Fijación del practicum metodológico en la continua perspectiva y análisis de la concepción diacrónica y sincrónica de la producción artística.

\begin{tabular}{|l|l|l|l|}
\hline Act. 4 & Recepción de dudas. & $\mathbf{5}^{\prime}$ & le \\
\hline
\end{tabular}

Objetivos: Con la recepción de dudas, al margen de su función, introduzco un paréntesis para acometer un nuevo lote de contenidos.

\begin{tabular}{|l|l|l|l|}
\hline Act. 5 & $\begin{array}{l}\text { ¿Concepto y forma? o ¿Forma y concepto? Lo apolíneo } \\
\text { y su canon de belleza: el desnudo masculino. } \\
\text { Escultura exenta: Kuroi y Korés. }\end{array}$ & 10 Pr & Pr \\
\hline
\end{tabular}

Ciclos de Mejora en el Aula (2020). Experiencias de Innovación Docente de la US Esta obra se distribuye con la licencia Creative Commons Reconocimiento-NoComercial-SinObraDerivada $\quad 4.0$ Internacional (CC BY-NC-ND 4.0.) 
¿Cómo o por qué pensáis llegaron los historiadores decimonónicos a adjudicar a las estatuas masculinas el calificativo de "Apolos"? ¿Quién es Apolo, qué identidad hereda del panteón egipcio? ¿Qué implica ser una deidad solar? ¿Se diseña/compone la forma que debe emitir un mensaje o se escoge una forma que se adapta al mensaje? ¿Se hace con conocimiento intelectual o por convencionalismo? ¿Eran estas figuras la prefiguración del dios Apolo? ¿Cuál es el principal mensaje que emiten? El Kuros como máxima expresión estética del mundo griego arcaico: quiénes eran los Kuroi/ Curetes en la mitología y culto, qué función tenían estas estatuas, dónde se ubicaban. Por último, se les introduce en los elementos técnicos y estéticos que revelan las claves estilísticas de su identificación y clasificación.

Objetivo: Reflexión sobre el proceso de adjudicar/crear un simbolismo estético e ideológico a un modelo artístico. Insistir en la dinámica de pervivencia y sincretismo de los códigos visuales.

\section{Act. 6 ¿Cómo se diferencian los Kurós?

Dadas las claves del vocabulario artístico se invita al alumno a analizar/ verificar lo aprendido sobre los principales ejemplares de los tres períodos: Arcaico antiguo.

Objetivos: Reafirmación de contenidos teóricos en la práctica visual analítica como verdadero aprendizaje. Asimilación de una estética plenamente relacionada con un contenido ideológico y su función. Visión de la escultura del período arcaico griego como producto que aúna el factor orientalizante con el autóctono griego. Aprendizaje del análisis visual a través de técnica y estilo.

Tabla 4 - Secuencia de actividades de la sesión cuarta

Sesión 4 - Grecia Clásica - Clásico Severo.

\begin{tabular}{|l|l|}
\hline Act. 1 & Contexto general del Período Clásico \\
\hline
\end{tabular} 15' $\mathrm{T}$

Contextualización, periodización y características de la escultura clásica: Estilo Severo, Clásico Pleno y Postclásico. Consagración del canon dórico. Nacimiento y desarrollo de los cánones de proporción, composición y movimiento: Contraposto y Diartrosis. Desnudo femenino de paños mojados.

\begin{tabular}{|c|c|c|c|}
\hline t. 2 & ece & 5 & le \\
\hline \multicolumn{4}{|c|}{ nprensión antes de introdu } \\
\hline (2) & 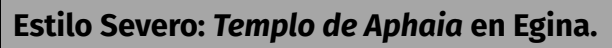 & & $\mathbf{T}$ \\
\hline \multicolumn{4}{|c|}{$\begin{array}{l}\text { Definición de las características del estilo severo } \\
\text { arcaico final al clásico pleno. Contextualización y } \\
\text { del estilo: cuestiones técnicas y compositivas. Arqu } \\
\text { de estilización máxima del templo dórico hexástilo, }\end{array}$} \\
\hline
\end{tabular}

Ciclos de Mejora en el Aula (2020). Experiencias de Innovación Docente de la US Esta obra se distribuye con la licencia Creative Commons Reconocimiento-NoComercial-SinObraDerivada Internacional (CC BY-NC-ND 4.0.) 
Análisis de los contenidos sobre las principales piezas originales conservadas en bronce.

Objetivo: Refuerzo de contenidos teóricos sobre la práctica analítica de las obras.

\begin{tabular}{|l|l|l|l|}
\hline Act. 5 & Relieve Severo - ¡El enigma Ludovisi! & 10 & AC \\
\hline
\end{tabular}

El análisis del llamado Trono Ludovisi, da pie para ejemplificar importantes cuestiones técnicas como el tratamiento de calidades. Así como iconográficas: ánodos vs. catábasis. Lluvia de preguntas: ¿Qué escena es esta, por qué? ¿Qué significa Afrodita? ¿Cómo nació? ¿Cómo suele componerse la escena del nacimiento de Afrodita? ¿Esta escena responde a la iconografía tradicional? ¿Por qué? ...

Objetivo: Aprendizaje de la observación analítica autónoma en los detalles técnicos e iconográficos que nos aportan cuestiones vitales para la identificación y clasificación de las obras de arte.

\section{\begin{tabular}{|l|l|l|l|}
\hline Act. 6 & ¡Are you ready, go! El Discóbolo y la contención del & 10 ' TP
\end{tabular} lanzamiento.}

¿Os parece clásica o severa? ¿Por qué? ¿Dónde aparecen novedades? Es el segundo ejemplar que estudiamos sobre el estudio anatómico en equilibrio y concentración máxima antes de lanzar ¿Conocemos alguno más a lo largo de la historia del arte?

objetivo: Nueva obra de transición. Aprender a mirar y a reconocer lo propio y lo nuevo.

Tabla 5 - Secuencia de actividades de la sesión quinta

\begin{tabular}{|c|c|c|c|}
\hline \multicolumn{4}{|c|}{ Sesión 5 - Grecia Clásica - Clásico Pleno } \\
\hline Act. 1 & Pericles y la reconstrucción de la Acrópolis - Grupo 1 & $20^{\prime}$ & $\mathbf{T}$ \\
\hline \multicolumn{4}{|c|}{$\begin{array}{l}\text { Trabajo de recopilación, síntesis y conclusión de un tema asignado } \\
\text { presentación del mismo con proyección simultánea. } \\
\text { Objetivo: Compromiso y desarrollo de la autonomía del aprendizaje. }\end{array}$} \\
\hline \multicolumn{4}{|c|}{ ¿Debatimos? } \\
\hline Act.2 & Interpelación directa. & $5^{\prime}$ & le \\
\hline \multicolumn{4}{|c|}{$\begin{array}{l}\text { Objetivo: Los estudiantes que asumen su participación deben prepararse } \\
\text { para resolver preguntas. }\end{array}$} \\
\hline Act. 3 & Recepción de dudas. & 5 & le \\
\hline \multicolumn{4}{|c|}{$\begin{array}{l}\text { Objetivo: Los estudiantes deben responsabilizarse de la asimilación del } \\
\text { contenido expuesto. }\end{array}$} \\
\hline Act. 4 & $\begin{array}{l}\text { Arquitectura: Fidias y la construcción del Partenón - } \\
\text { Grupo } 2 .\end{array}$ & $20^{\prime}$ & $\mathbf{T}$ \\
\hline
\end{tabular}

Ciclos de Mejora en el Aula (2020). Experiencias de Innovación Docente de la US Esta obra se distribuye con la licencia Creative Commons Reconocimiento-NoComercial-SinObraDerivada $\quad 4.0$ Internacional (CC BY-NC-ND 4.0.) 
Trabajo de recopilación, síntesis y conclusión de un tema asignado y presentación del mismo con proyección simultánea.

Objetivo: Compromiso y desarrollo de la autonomía de aprendizaje.

\begin{tabular}{|l|l|l|l|}
\hline \multicolumn{5}{|c|}{ ¿Debatimos? } \\
\hline Act. 5 & Interpelación directa. & $\mathbf{5}^{\prime}$ & le \\
\hline
\end{tabular}

Objetivo: Los estudiantes que asumen su participación deben prepararse para resolver preguntas.

\begin{tabular}{l|l|l|l|}
\hline Act. $\mathbf{6}$ & Recepción de dudas. & $\mathbf{5}^{\prime}$ & le \\
\hline $\begin{array}{l}\text { Objetivo: Los estudiantes deben responsabilizarse de la asimilación del } \\
\text { contenido expuesto. }\end{array}$
\end{tabular}

Tabla 6 - Secuencia de actividades de la sesión sexta

\begin{tabular}{|l|l|l|l|}
\hline \multicolumn{4}{|c|}{ Sesión 6 - Grecia Clásica - Clásico Pleno } \\
\hline Act. 1 & $\begin{array}{l}\text { Recepción clase anterior sobre Arquitectura } \\
\text { Partenón. }\end{array}$ & $1^{\prime}$ & C \\
\hline
\end{tabular}
de los alumnos y complementación si fuera necesario: Función política de la Acrópolis de Pericles. Consagración del canon dórico: Octástilo, períptero, anfipróstilo en el Partenón. Correcciones ópticas, etc.

\begin{tabular}{|l|l|l|l|}
\hline Act. 2 & $\begin{array}{l}\text { Escultura: Fidias y el programa iconográfico del } \\
\text { Partenón. }\end{array}$ & $\mathbf{4 0}$ & TP \\
\hline
\end{tabular}

El estilo "Partenón": Metopas/Frontones/Friso jónico. Atenea Partenos, Promachos y Lemnia.

Objetivo: Visión general de la producción escultórica en relieve y exenta para aprender cómo se gesta la constitución de un estilo o escuela, ligada siempre a un gran maestro y una empresa política. Estudio iconográfico de los distintos repertorios figurativos.

\begin{tabular}{|l|l|l|l|}
\hline Act. $\mathbf{3}$ & Recepción de dudas. & $\mathbf{5}^{\prime}$ & le \\
\hline $\begin{array}{l}\text { Objetivo: Finalización y afianzamiento de la comprensión de los contenidos } \\
\text { directos sobre el Partenón. }\end{array}$
\end{tabular}

Tabla 7 - Secuencia de actividades de la sesión séptima

\section{Sesión 7 - Grecia Clásica - Clásico Pleno.}

\section{\begin{tabular}{|l|l|l|l|} 
Act. 1 & El urbanismo a las puertas de la acrópolis: Los Propileos. & 15' & TP \\
\hline
\end{tabular}}

Actividad teórico-práctica sobre el diseño de Mnesiclés.

Objetivo: Reflexión sobre la función organizadora, estética y política del urbanismo.

\begin{tabular}{|l|l|l|l|}
\hline Act. 2 & $\begin{array}{l}\text { Arquitectura: El último estilo clásico: Estilo Bello en la } \\
\text { Acrópolis. }\end{array}$ & $15 ’$ & TP \\
\hline
\end{tabular}

Ciclos de Mejora en el Aula (2020). Experiencias de Innovación Docente de la US Esta obra se distribuye con la licencia Creative Commons Reconocimiento-NoComercial-SinObraDerivada $\quad 4.0$ Internacional (CC BY-NC-ND 4.0.) 
Actividad teórico-práctica sobre la diversidad de estilos en la acrópolis: orden jónico y el estilo bello en dos templos: El Erection y el templo de Atenea Niké. Objetivo: Reflexión sobre la visión histórico-artística plana de algunos complejos arquitectónicos con hitos icónicos dominantes.

\begin{tabular}{|l|lc|l|l|}
\hline Act. 3 & Escultura: ¿Quién fue Policleto? ¿Qué hizo? ¿Cómo lo hizo? & 10 ' & Tr \\
\hline
\end{tabular}

El día anterior avisaré que voy a hablar de Policleto. Solicitaré que cada alumno extraiga información sobre el asunto y comenzaremos dando la palabra uno a uno.

Objetivo: Refuerzo de los contenidos teóricos a través de la propia tarea de documentación.

\begin{tabular}{|l|l|l|l|}
\hline Act. 4 & $\begin{array}{l}\text { El canon de proporciones masculino: Policleto y el } \\
\text { Doriforo. }\end{array}$ & $10^{\prime}$ & C \\
\hline
\end{tabular}

Cierre de los contenidos de la actividad anterior a través de un ejercicio de conclusiones que ordenen la estructura de los datos aportados por todos: alumnos y profesora.

Objetivo: Conclusiones sobre Grandes empresas, grandes maestros. Su colaboración e influencias.

\begin{tabular}{l|l|l|l|}
\hline Act. 5 & $\begin{array}{l}\text { ¿Qué obra es esta? ¿Quién la hizo? ¿Dónde la hizo? } \\
\text { ¿Dónde está el canon? ¿Cómo lo realiza? ¿Dónde está la } \\
\text { influencia de Fidias? }\end{array}$ & 10 TP \\
\hline
\end{tabular}

Prueba práctica voluntaria de análisis técnico y estilístico sobre otra de las obras maestras de Policleto: El Diadumeno, a partir de estas las preguntas articuladoras del método científico.

Objetivo: Fomento de la participación. Refuerzo de contenidos y de las actitudes del practicum.

Tabla 8 - Secuencia de actividades de la sesión octava

\begin{tabular}{|l|l|l|l|}
\hline \multicolumn{4}{|c|}{ Sesión 8 - Grecia Postclásica o Crisis del siglo IV. } \\
\hline Act. $\mathbf{1}$ & $\begin{array}{l}\text { Escultura: Los sucesores de Fidias y Policleto. El } \\
\text { desbordamiento del canon. }\end{array}$ & $\mathbf{1 5}^{\prime}$ & $\mathbf{T}$ \\
\hline $\begin{array}{l}\text { Contexto general de las coordenadas ideológico-culturales de la gran crisis } \\
\text { que marca el siglo final del período clásico } \\
\text { Objetivo: Observación del reajuste en el cambio de mentalidades y la } \\
\text { inmediata respuesta artística. }\end{array}$ & $\mathbf{1 5}$ & TP \\
\hline Act. $\mathbf{2}$ & Práxiteles - Lirismo y gracia femenina (charis). \\
\hline $\begin{array}{l}\text { Características principales de su estilo: La sensualidad de la curva } \\
\text { praxiteliana en el Hermes y Dionisos niño. La charis de la Afrodita de Cnido. } \\
\text { Objetivo: Observación de los contenidos teóricos verificados en las obras } \\
\text { artísticas. }\end{array}$ & $\mathbf{1 0}$ & TP \\
\hline Act. $\mathbf{3}$ & Scopas - La pulsión patética &
\end{tabular}

Ciclos de Mejora en el Aula (2020). Experiencias de Innovación Docente de la US Esta obra se distribuye con la licencia Creative Commons Reconocimiento-NoComercial-SinObraDerivada $\quad 4.0$ Internacional (CC BY-NC-ND 4.0.) 
Características principales de su estilo a través del análisis del pathos de su Ménade danzante.

Objetivo: Confrontación de los métodos de expresión. Contrapunto a la charis praxiteliana.

\section{Act. 4 Lisipo - El último clásico, la estilización del canon} policlético.

Características principales del estilo clasicista más ortodoxo del siglo IV a través de su Apoxiomenos, desbordando el canon a través de su estilización y mayor profundidad espacial.

Objetivo: Confrontación del proceso evolutivo del estilo imperante hasta su superación.

\section{Act. 5}

¿Lisipo? ¿El último clásico? ¿En serio?

Presentación sin información previa de la copia romana en mármol del artista griego Glikon para las Termas de Caracalla, conocido como el Hércules Farnesio, sobre el original en bronce de Lisipo.

¿Es objetiva la genealogía artística de una copia? ¿Es rigurosa la copia como fuente documental? La bipolaridad estética sostenida por el virtuosismo artístico del maestro ¿Es libre el artista a la demanda del mercado?... Obra que presenta la estética del próximo y último periodo griego: El Helenismo. Objetivo: Invitación al debate y la reflexión sobre la autoría, el estilo, etc.

\section{Cuestionario inicial/final.}

Como se indica desde la coordinación del curso, el cuestionario debe diseñarse para que revele los conocimientos previos de los alumnos sobre los contenidos capitales, para resolver los obstáculos de aprendizaje con las herramientas metodológicas y para evaluar el resultado de la aplicación del CIMA. Se diseñó un cuestionario de siete preguntas que alternaban enunciados de planteamientos previos y preguntas más o menos directas sobre la materia. Las posibles respuestas debían evidenciar el nivel de conocimiento transversal en su contexto estético, técnico, iconográfico-iconológico y compositivo. También los estereotipos adquiridos sobre la cultura y el nivel de conocimiento de los grandes períodos: prehelénico, arcaico y clásico. Se presenta a continuación.

Ciclos de Mejora en el Aula (2020). Experiencias de Innovación Docente de la US Esta obra se distribuye con la licencia Creative Commons 
Este cuestionario es una prueba de nivel de conocimiento para aplicar un correcto punto de partida en nuestro aprendizaje. No es puntuable. Al finalizar el tema, volveremos a contestarlo para observar nuestra evolución. Por favor, contesta desde tu conocimiento y no busques o consultes datos al respecto.

1. Si pasearas por la Acrópolis de Atenas ¿Reconocerías distintos estilos artísticos? ¿En qué te basarías para diferenciarlos? Objetivo: Reconocimiento de los tres órdenes griegos básicos: dórico, jónico y corintio. Distinta tipología arquitectónica, etc.

2. El bufete que representa al gobierno griego te solicita como historiador del arte un argumento de peso para justificar ante el Tribunal Internacional de La Haya la propuesta que va a plantear para llegar a un acuerdo con Inglaterra y zanjar el caso: Recuperar los mármoles del Partenón, a cambio de no reclamar más otras piezas griegas. ¿Qué argumentos esgrimirias? ¿Y si fueras uno de los miembros del Tribunal por qué considerarías que la propuesta es aceptable, o no? Objetivo: Nivel de conocimiento del Partenón y su contexto.

3. Cuando paseas por la ciudad podemos observar una interesante tradición artística griega entre nosotros, ¿puedes decirme dos ejemplos? Objetivo: Reconocimiento Legado griego.

4. ¿Entre toda la mitología griega, sabrías identificar la identidad de un personaje o de una deidad? ¿cómo lo identificas? ¿Conoces el significado de esos elementos? Objetivo: Nivel de conocimiento sobre Iconografía-iconología griega.

5. En escultura, ¿podrías reconocer como griegas dos piezas de dos estilos diferentes? ¿En qué y por qué los distinguirías: composición, expresión, materiales, etc.? Objetivo: Reconocimiento de los estilos principales: arcaico, clásico... 
6. El satélite europeo Eurostar V se dirige hacia el planeta Marte para su colonización. Su tripulación lleva una cápsula del tiempo para futuras civilizaciones marcianas con pequeñas piezas representativas de los países del Viejo Continente. Francia, desde luego, la Torre Eiffel. ¿Cuál debería ser la griega, por qué? Objetivo: Indagación de estereotipos adquiridos.

7. Eres el maquetador de una editorial y preparas la portada de la novela Ulises entre sirenas. ¿Cuál/es sería la obra de arte griego que te viene a la mente para contextualizar cronológicamente con el personaje y su cultura? Objetivo: Conocimiento arte prehelénico.

\section{Aplicación del CIMA}

El CIMA se inició con una caída del sistema de veinticinco minutos que me impidió entrar en la plataforma. Los alumnos mostraron un buen talante, esperando pacientemente y pude comenzar la sesión en la que debí sintetizar todo el proceso. En esta sesión pude realizar las Actividades 1, 2, 4 y 7. Posponiendo la Actividad 3 a la siguiente. Los alumnos más activos habitualmente, tomaron la iniciativa y gracias a ellos fue posible construir la secuencia preparada, a la que se fueron sumando tímidamente por chat algún compañero más.

Tras superar el percance informático, el resto del CIMA se ha desarrollado en un clima de trabajo adecuado, con los alumnos colaboradores, apoyando el ciclo y comportándose con total normalidad, pero sin ser excesivamente expresivos a micro abierto. El nivel de integración y participación en las actividades ha ido paulatinamente en aumento conforme han comprobado la finalidad didáctica de las actividades y han entendido que la participación no implica contestar correctamente, sino aprender a aprender, contrastando analíticamente los conocimientos Ciclos de Mejora en el Aula (2020). Experiencias de Innovación Docente de la US
Esta obra se distribuye con la licencia Creative Commons 
previos con los que se están exponiendo en clase. También, porque conforme nos hemos ido acercando a la Grecia Clásica reconocen los estereotipos adquiridos como el Partenón y, sobre todo, del concepto de belleza que tienen asimilado y al reaccionan sensiblemente.

Precisamente, por ello, en los ejercicios que han desmontado sus esquemas, es decir, las Actividades de Contraste, han sido más activos. El impacto de descubrir algo nuevo en un conocimiento que creen establecido promueve un factor sorpresa que es vital para estimularlos. Visualizar hasta dónde se pueden remontar los ancestros icónicos que nos representan hoy día como cultura o país (ibérico/toro). Descubrir que detrás de estas obras, se encuentra todo un sistema de ordenación filosófica, religiosa y política que es la base de nuestra cultura colectiva. O que para hacer un templo perfecto hay que hacerlo perfectamente imperfecto, evidencia el proceso de reelaboración que se produce en sus mentes y el nuevo peldaño ascendido.

Valoro los avances en relación a mi conexión emocional con mis alumnos. Como he mencionado, la emoción colectiva, como ejercicio de contemplación estética, se ha producido en la cuarta sesión. Tras la rigidez arcaica, el impacto de la belleza de los bronces severos clásicos, últimos exponentes originales, ha sido general. La llamada de atención sobre la última oportunidad de contemplar la genuina escultura clásica griega en bronce y el factor de pérdida que supone estudiarlas a través de las copias romanas, ha ayudado a elevar el clima respecto al milagro de la genialidad artística humana como máxima cota de expresión. Lo que es importantísimo para cultivar sus sentidos, para motivar al alumno en la disciplina y alimentar el espíritu para conectar, interactuar y construir como una argamasa el proceso de aprendizaje con la emoción (Porlán, 2020a). Durante el CIMA, los chats de despedida eran más expresivos: "Qué clase más interesante" o "Una clase muy interesante la de hoy". 
La mayor dificultad la he encontrado en la convocatoria de participación voluntaria en las actividades de clase. Sólo un alumno la ha aceptado como una gran experiencia y con enormemente gratitud. En el momento que cedí la palabra seis alumnos abandonaron la plataforma. El resto de compañeros continuó en clase, pero no interactuaron en ningún momento a pesar de que les animé a ello, por lo que el debate no pudo darse. El compromiso del estudiante con su formación es una cuestión capital que debe atajarse en todos los ciclos formativos. Al hilo de lo anterior, la participación en la evaluación de aprendizaje revela la misma falta de compromiso sólo 13 alumnos han realizado la encuesta inicial y final.

El nivel general era bajo, como se preveía, pues no pertenecen al Grado de Historia del Arte. En el registro más bajo de conocimiento, el obstáculo principal que se aprecia es que se verifica un desinterés personal por la materia. Salvo en la Pregunta 7 (Fig. 4) que partía de un desconocimiento general sobre el arte prehelénico y su resultado ha sido necesaria y evidentemente evolutivo y la Pregunta 6 (Fig. 4) sobre el estereotipo Grecia/Partenón que se muestra como una foto fija, las respuestas de cada nivel mantienen un margen de evolución del $10 \%$ de media entre la encuesta inicial y la final. En el nivel más bajo, cuando ésta se produce, se proyecta directamente sobre el siguiente segmento, el inicial, pero en otras, que ejemplifica la Pregunta 2 (Fig. 4), saltan al intermedio, dando un paso cualitativo en la evolución del aprendizaje. El segmento formado por el nivel inicial y el intermedio constituyen el de mayor tránsito. Siendo el intermedio el que recibe todo el proceso de evolución y se muestra como el techo de cristal que hay que romper. Se constata la estabilidad evolutiva del $10 \%$ en el nivel alto entre la encuesta inicial y la final, incluso cuando parte de un $0 \%$ en la evaluación inicial como la Pregunta 7. Sin embargo, en la Pregunta 1 (Fig. 4) que constituye el bastión del tema, el aumento que recibe el nivel más alto proviene directamente de los más bajos, produciéndose la máxima superación del obstáculo. 


\section{Pregunta 1.}

Si pasearas por la Acrópolis de Atenas ¿Reconocerías distintos estilos artísticos? ¿En qué te basarías para diferenciarlos?

Conocimiento de los tres órdenes griegos: dórico, jónico, corintio y su distinta tipología arquitectónica.

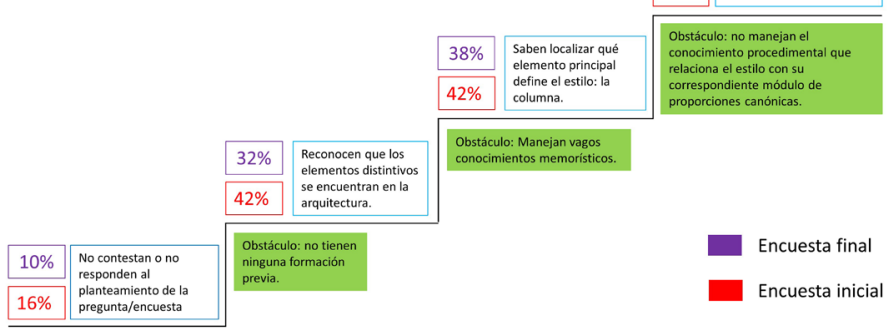

\section{Pregunta 2.}

El bufete que representa al gobierno griego te solicita como historiador del arte un argumento de peso para justificar ante el Tribunal Internacional de La Haya la propuesta que va a plantear para llegar a un acuerdo con Inglaterra y zanjar el caso: Recuperar los mármoles del Partenón, a cambio de no reclamar más otras piezas griegas. ¿Qué argumentos esgrimirías? ¿Y si fueras uno de los miembros del Tribunal por qué considerarías que la propuesta es aceptable, o no?

Conocimiento del concepto de Patrimonio cultural material: El Partenón y su contexto.

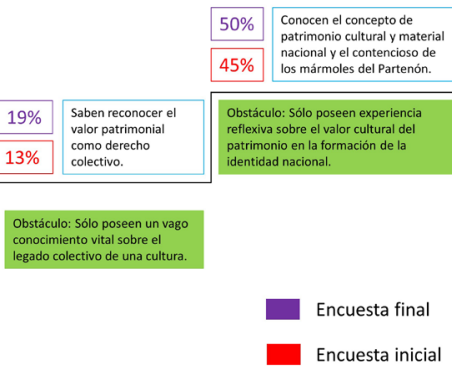

\section{Pregunta 6.}

El satélite europeo Eurostar V se dirige hacia el planeta Marte para su colonización. Su tripulación lleva una cápsula del tiempo para futuras civilizaciones marcianas con pequeñas piezas representativas de los paises del Viejo Continente. Francia, desde luego, la Torre Eiffel.

¿Cuál debería ser la griega, por qué?

Indagación de estereotipos adquiridos.

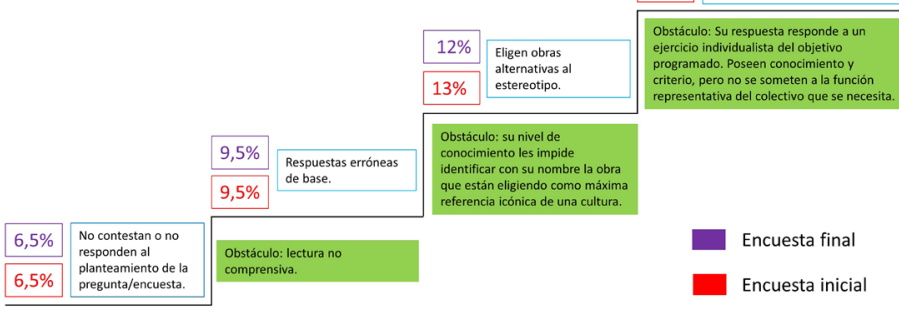

Ciclos de Mejora en el Aula (2020). Experiencias de Innovación Docente de la US (c) E) Esta obra se distribuye con la licencia Creative Commons 


\section{Pregunta 7.}

Eres el maquetador de una editorial y preparas la portada de la novela Ulises entre sirenas. ¿Cuál/es sería la obra de arte griego que te viene a la mente para contextualizar cronológicamente con el personaje y su cultura?

Conocimiento sobre el arte prehelénico.

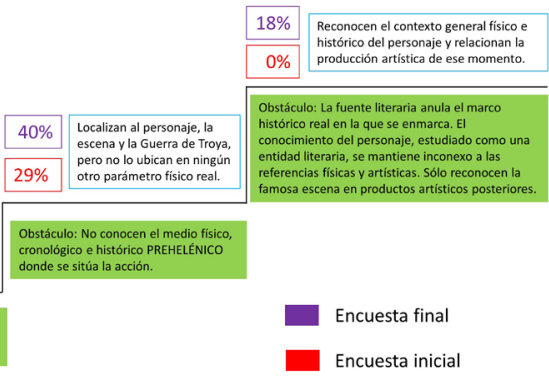

Figura 4. Escalera de evaluación: preguntas 1, 2, 6 y 7

\section{Evaluación del CIMA puesto en práctica.}

\section{Cuestiones a mantener y cambios a introducir para un futuro Ciclo de Mejora.}

El conocimiento de los modelos mentales con los que el alumno se enfrenta al aprendizaje me revela la necesidad de mantener, no sólo ésta, sino todas las herramientas utilizadas. Cada una de ellas ha demostrado su utilidad y la necesidad de una aplicación metodológicamente didáctica a través de la interacción de las mismas con su correspondiente reflejo en el método de evaluación. Destacaría entre todas ellas las Actividades de Contraste que, en un grupo ajeno, en principio, a los intereses de la asignatura, son los que dinamizan más al alumno emocionalmente y lo mantienen alerta y participativo durante la clase.

Al hilo de la participación, he propuesto en la metodología una cooperación voluntaria de mayor peso del alumnado, esperando y valorando el compromiso con su aprendizaje, pero mis expectativas no se han visto materializadas. Tampoco, del todo, en la secuencia de actividades Ciclos de Mejora en el Aula (2020). Experiencias de Innovación Docente de la US
Esta obra se distribuye con la licencia Creative Commons 
teóricas que diseñada en una situación ideal de participación y dinamismo que después no se verifica, consumiendo mucho tiempo e impiden finalizar el CIMA. Si la materia que se ajusta para que dé tiempo la aplicación de la secuencia de actividades no se repone con el trabajo independiente del alumno, el sistema no cubre los conocimientos básicos.

\section{Aspectos de la experiencia que se incorporan a la práctica docente y principios didácticos que deben permanecer en el futuro.}

Me quedo con mi entusiasmo a la hora de crear actividades de contraste que les resulten atrayentes y les dirija hacia el descubrimiento de un nuevo mundo de conocimiento (Bain, 2007 y Finkel, 2008) y mis expectativas con la posibilidad de que, se entusiasmen y descubran la elevación y el goce que produce la contemplación estética. Porque eso les llevará a buscar por si mismos todas las respuestas a esas preguntas capitales que gestionan el aprendizaje.

Para el curso que viene y, en general, en mi docencia futura seguiré utilizando con el modelo metodológico intermedio. Utilizaré el cuestionario para evaluar el nivel de conocimientos de alumnado y el modelo mental sobre la materia al que responden. Con ello, podré depurar el mapa de contenidos, cuya estructura basada en preguntas capitales mantengo, y también la secuencia de actividades a sus necesidades concretas y a tiempo real. Dejaré exclusivamente los ejercicios que mejor funcionaron el curso anterior, atendiendo a mi autoevaluación y a la evaluación de mi docencia de los alumnos y diseñaré y experimentaré el resultado de otros nuevos específicos según el resultado de la evaluación de nivel del curso actual. Para reforzar la participación del alumnado en mi sistema metodológico,

Ciclos de Mejora en el Aula (2020). Experiencias de Innovación Docente de la US Esta obra se distribuye con la licencia Creative Commons 
incluiré la participación en todas las actividades que se incorporen dentro de la evaluación con su correspondiente gratificación. Mantendré la posibilidad de realizar el trabajo de curso de forma voluntaria, apoyado en mi tutoría como medio de canalización de su zona de desarrollo propio, para que aprendan a construir su personal sistema de cimentación del conocimiento real adquirido de modo maduro e independiente (Vygotsky, 2002 y Bruner, 1978).

Ciclos de Mejora en el Aula (2020). Experiencias de Innovación Docente de la US Esta obra se distribuye con la licencia Creative Commons 
Palabras clave: Arte Universal, Grado de Historia, Docencia Universitaria, Experimentación Docente Universitaria.

Keywords: Universal Art, Degree in History, University Teaching, University Teaching Experimentation.

\section{Referencias bibliográficas}

Bain, K. (2007). Lo que hacen los mejores profesores universitarios. Valencia: Universitat de València.

Bruner, J., Goodnow, J., Austin, G. y Vegas, J. (1978). El proceso mental en el aprendizaje. Madrid: Narcea.

Finkel, D. (2008). Dar clase con la boca cerrada. Valencia: Universitat de València.

Piaget, J. (1970). Educación e instrucción. Buenos Aires: Proteo.

Porlán, R. (2019). Enseñanza universitaria. Cómo mejorarla. Madrid: Morata.

Porlán, R. (2020a). El cambio de la enseñanza y el aprendizaje en tiempos de pandemia. Revista de Educación Ambiental y Sostenibilidad 2 (1), 1502-1 - 1502-7.

Porlán, R. (2020b). Docentes universitarios. Una formación centrada en la práctica. Madrid: Morata.

Vygotsky, L. (2003). El desarrollo de los procesos psicológicos superiores. Barcelona: Crítica.

Ciclos de Mejora en el Aula (2020). Experiencias de Innovación Docente de la US Esta obra se distribuye con la licencia Creative Commons 